\title{
In vivo evidence of htid suppressive activity on ErbB-2 in breast cancers over expressing the receptor
}

\author{
Ursula Kurzik-Dumke*+1, Manuela Hörner ${ }^{1}$, Maria R Nicotra², Michael Koslowski and Pier G Natalił4
}

\begin{abstract}
Background: Htid encoded proteins are physiological partners of a wide spectrum of molecules relevant to neoplastic transformation. One of the molecular ligands of the cytosolic hTid-L and hTid-I forms is the ErbB-2 receptor variably over expressed in diverse solid tumors. Altered ErbB-2 signalling is associated with an unfavourable prognosis in about $30 \%$ of human breast malignancies.

Methods: We evaluated htid and HER-2 expression by quantitative real time PCR in tumors of different TNMG status and by immunohistochemistry in a cohort of breast tumors of the Luminal A, B, HER-2 and triple negative subtype.

Results: The RT-PCR analysis revealed that aberrant expression of all three htid forms correlates with malignant transformation. Furthermore, elevated hTid-L expression can be associated with less aggressive tumors. The immunohistochemical testing revealed that tumors of the luminal A subtype are characterized by a high level of htid ( $81 \%$ ). In contrast htid expression is significantly lower in tumors of the Luminal B (20\%) and HER-2 (18\%) subtype over expressing the receptor and in the triple negative (40\%) more aggressive malignancies. A statistically significant inverse correlation between htid and ErbB-2 expression was found in human breast $(p<0,0001)$ and non-mammary tumors $(p<$ $0,007)$, and in transgenic mice carrying the rat HER-2/neu oncogene.
\end{abstract}

Conclusions: Our findings provide in vivo evidence that htid is a tissue independent and evolutionarily conserved suppressor of ErbB-2.

\section{Background}

Htid-1 is the human counterpart [1] of the Drosophila melanogaster lethal(2)tumorous imaginal discs (l(2)tid) tumor suppressor gene [2]. (In the following we omit the number "1" in the designation of the gene because it suggests the presence of a further copy in the genome. As sequencing of the human, mouse and Drosophila genomes revealed only one tid gene is present in all these species.)

The tid genes encode members of the highly conserved DNAJA3 protein family acting as Hsp70/Hsc70 co-chaperones [3-5]. In the fly, defined recessive mutations have been shown to be oncogenic in cell lineages responsive to

*Correspondence: kurzik@uni-mainz.de

1 Institute of Medical Microbiology and Hygiene, Comparative Tumor Biology Group, University Medical Center, Johannes Gutenberg University, Obere

Zahlbacher Str. 63, 55131 Mainz, Germany

+ Contributed equally

Full list of author information is available at the end of the article the signalling mediated by Patched (Ptc), the receptor for the Hedgehog $(\mathrm{Hh})$ signalling molecule [6]. The physiological Tid-Ptc binding is conserved in the fly, in the mouse and in man [6,7]. Using the yeast two-hybrid technique $\mathrm{h} t i d$ has been isolated as a molecular partner of a variety of tumor related proteins [6-15] including receptor tyrosine kinases (RTK) such as TRK-A [14] and ErbB2 [15] of the epidermal growth factor receptor (EGFR) family comprising four receptors (ErbB1-4) [16]. As for other members of these receptors, the ErbB-2 signal output is controlled by a dynamic equilibrium between the on/off states. In this regard, in vitro studies have shown that interaction of the cytosolic hTid-L and hTid-I proteins $[1,7]$ with ErbB-2 promotes ubiquitination and degradation of the receptor resulting in down regulation of its signalling thus, of its oncogenic potential/activities [15]. (The aforementioned hTid-I form, 453 amino acids in size [1,7] is designated by Kim et al. [15] as hTid-S.). 
This is of interest since over expression of ErbB-2, often caused by gene amplification, characterizes $20-30 \%$ of human breast cancers being casually linked to an aggressive clinical course of these tumors [17] and a variable percentage of extra-mammary tumors [18]. These data are consistent with the biology of the EGFR family members mediating control of cellular responses such as proliferation, differentiation, survival and apoptosis, essential for the maintenance of the transformed and metastatic phenotype. Thus, in the context of the functional link between the htid encoded proteins and ErbB-2 in the present study we addressed the question whether in human sporadic breast tumors the in vivo expression profiles of the two tumor genes provide support for htid function as a negative regulator of ErbB-2 activity. Since htid encodes three splice forms [1,7] we compared their expression levels with that of the HER-2 transcript using quantitative real-time PCR (RT-PCR) and the comparative $\mathrm{Ct}$ method for relative quantification [19] in a panel of human breast tumors of diverse differentiation grades $[20,21]$. Furthermore, we performed an extensive phenotypic comparative analysis of the expression of the two target genes in a panel of human breast cancers classified according to Sotiriou and Pusztai [22] as the subtypes luminal A, luminal B, HER-2 and triple negative, and by non breast tumors over expressing ErbB-2. Furthermore, since the identification of an experimental in vivo model mimicking human breast tumors is of great interest to dissect the oncosuppressive activity of the tid gene at the molecular level, we investigated mammary malignancies in transgenic mice carrying the rat counterpart of HER-2/ neu. The data gained by RT-PCR showed that the single htid splice forms [1,7] are differentially expressed in normal mammary epithelium. Whereas the htid-I form shows the highest expression level $\left(2^{-\Delta \mathrm{Ct}}=0,50\right)$, the htid$\mathrm{L}$ form expression level is about 7 times lower $\left(2^{-\Delta \mathrm{Ct}}=\right.$ $0,07)$. The htid-S form is present only residually $\left(2^{-\Delta \mathrm{Ct}}=\right.$ $\left.3,18 \times 10^{-5}\right)$. Generally, the expression level of each of the single htid splice forms is lower as that determined for $H E R-2\left(2^{-\Delta C t}=4,99\right)$. In breast tumors the expression levels of all three htid transcripts are altered. As a result the amount-ratio among the diverse htid forms and the HER2 transcript changes drastically.

The phenotypic analysis of tumor specimens demonstrated that hTid and ErbB-2 expression are inversely correlated in breast cancer either primary $(\mathrm{p}<0.0001)$ or metastatic ( $\mathrm{p}<0.023)$, in primary non-breast tumors $(\mathrm{p}<$ 0,007 ) and in breast tumors generated in HER-2/neu transgenic mice. Overall these results identify htid as a novel negative modulator of $H E R-2$, suggesting that strategies capable of increasing or stabilizing its cellular level may result into a decrease of the oncogenic signalling mediated by the receptor.

\section{Materials and methods Patients and tissues}

The specimens of breast and non breast tumors employed in this study originate from patients free from therapy undergoing treatment at various institutions. Normal breast tissue was obtained from cosmetic mammoplast. Samples used for RT-PCR originate from archive of tumor samples kindly provided by Dr. Ö. Türeci of the Experimental and Translational Oncology III, University Medical Center, Johannes Gutenberg University, Mainz. Specimens assayed by immunohistochemistry were obtained at the "Regina Elena" Cancer Institute, Rome, Italy. The patients consented to the experimental use of the specimens as requested by the Institutional Ethical Committee (IRE resolution of $n^{\circ} 7$ of July $2^{\text {nd }}$, 2003 adjourned on January, $1^{\text {st }}, 2006$ ). All tumor samples used in this study were characterized with respect to their TNMG status according to WHO specifications following the criteria of the International Union Against Cancer UICC [20] and the Elston and Ellis method [21]. Freshly collected tissue samples were divided into two parts. One part was processed for routine histopathological examination, the other was immediately shock frozen. The collected samples were stained with $1 \%$ toluidine blue to monitor morphology and the ratio of glandular epithelium and interstitium. Exclusively samples characterized by high amount of glandular epithelium, over $80 \%$, were used for RT-PCR and immunohistochemistry. Non-consecutive $4 \mu \mathrm{m}$ thick cryostat sections were prepared, fixed for $10 \mathrm{~min}$ in absolute acetone, and either submitted to indirect immunoperoxidase (IPP) staining or stored at $20^{\circ} \mathrm{C}$ over a period of a month with no loss of immune reactivity. The immunohistochemical analysis was performed on 75 randomly selected cases of infiltrating ductal carcinomas and 30 metastatic (25 lymphathic and 5 extra-lymphatic lesions) carcinomas building the first cohort. The second cohort of specimens consisted of 58 primary infiltrating breast tumors classified according to Sotiriou and Pusztaim [22] into the four distinct subtypes: luminal A, luminal B, HER-2 and triple negative on the basis of the results of immunohistochemical evaluation of expression of the estrogen and progesterone hormone receptors and the ErbB-2 receptor kinase, cytokeratins and the proliferation marker Ki67. This cohort encompassed a total of 24 tumors over expressing ErbB-2 (7 cases classified as luminal B and 17 cases of the HER-2 subtype), and a total of 34 ErbB-2 negative tumors (8 luminal B, 16 luminal A cases and 10 triple negative tumors). Non breast tumors were represented by a selected panel of 18 malignancies including thyroid, colon, ovarian and renal carcinomas. Experimental breast tumors developed during a period of 22 and 30 weeks in transgenic Balb/c mice carrying the rat HER-2/neu onco- 
gene [23] were kindly provided by Prof. Guido Forni (Dept. of Clinical and Biological Sciences, Univ. of Turin, Italy) and processed like the human specimens.

\section{Antisera}

Rabbit anti-hTid antiserum recognizing both the human and the mouse Tid proteins was generated, purified and characterized as previously described $[6,7,13]$. The rabbit polyclonal antiserum (AO 485) to human ErbB-2 cross reacting with the rat receptor was obtained from DAKO Cytomation (Denmark). The polyclonal antibody raised in chickens (GTX14027) recognizing the rat receptor was obtained from Gene Tex, Inc. (S. Antonio, TX. USA). Alexa fluor 594-labelled goat anti-rabbit IgG antiserum was purchased from Molecular Probes (Eugene, OR. USA). Fluorescein (FITC)-labelled rabbit anti chicken IgG was purchased from Sigma (St. Louis MA, USA).

\section{Immunohistochemical staining}

Indirect immunoperoxidase staining of frozen tissue sections was performed according to standard procedures using the Vectastain ABC kit (Vector Labor, Burlingame, CA). Anti-hTid $[6,7,13]$ was used at a concentration of 10 $\mu \mathrm{g} / \mathrm{ml}$ while the anti ErbB-2 antibody (AO485) was employed as suggested by the manufacturer. Sections were counterstained using Mayer's hematoxylin. Positive controls were represented by archival specimens of known expression of both antigens, while negative controls were represented by sections incubated with normal rabbit immunoglobulins or isotype matched mouse imnunoglobulins. The evaluation of the staining was performed as follows: htid was scored negative when cytoplasmic staining was absent or very weak and positive when a homogeneous granular cytoplasmic staining, ranging from moderate $(+)$ to strong $(2+/ 3+)$, was detected. ErbB-2 expression was established on the basis of a strong $3+$ cell membrane staining or a $2+$ staining intensity with a positive FISH assay [24]. Scoring of the expression of the antigens investigated was performed independently by two pathologists. Double immunofluorescence staining of breast tumors developed in HER-2/ $n e u$ transgenic mice was performed by incubating the tumor sections at first with the rabbit anti-hTid antiserum and Texas Red labelled anti rabbit IgG. After blocking with decomplemented normal rabbit serum at a dilution of $1: 100,30 \mathrm{~min}$, the sections were stained with chicken anti rat HER-2 antibody and the FITC-labelled rabbit anti chicken IgG. Sections were evaluated employing a Leica DMIRE2 microscope equipped with a Leica DFC 350FX camera and elaborated by a Leica FW4000 deconvolution software (Leica, Solms, Germany).

\section{RNA isolation, RT-PCR, and quantitative real-time RT-PCR}

To generate cDNAs corresponding to the transcripts encoded by the target genes investigated extraction of total cellular RNA was performed using the Oligotex RNeasy Mini Kit (Qiagen, Hilden, Germany) and reversetranscription with Superscript II (Invitrogen, Heidelberg, Germany). The integrity of the cDNAs generated was investigated by amplification of p53 transcripts, using the primers 5'-CGT GAG CGC TTC GAG ATG TCC G-3' (sense) and 5'-CCT AAC CAG CTG CCC AAC TGT AG-3' (antisense) as described previously [13]. For end point RT-PCR analysis of individual transcripts $0.5 \mu \mathrm{l}$ first-strand cDNA were amplified using the QuantiTect SYBR Green PCR Kit (Qiagen), transcript-specific oligonucleotides (300 $\mathrm{nM}$ each) and $1 \mathrm{U}$ HotStarTaq DNA polymerase (Qiagen) in a $30 \mu$ reaction, 40 cycles, in accordance with the manufacturer's instructions. Each PCR reaction was performed in triplicates using the following reaction conditions: initial denaturation/activation for 15 minutes at $95^{\circ} \mathrm{C}, 30$ seconds at $94^{\circ} \mathrm{C}, 30$ seconds of annealing, and 30 seconds at $72^{\circ} \mathrm{C}$. A template-free negative control was included in each experiment. To amplify fragments corresponding to a defined htid splice-form $(1,13)$, the following primer combinations were used: htid-L, 5'-GTT GAC ATT CAA TCA AGC TGC-3'(sense) and 5'-CTG GGA TAT CAT GAG GTA AAC-3'(antisense), htid-I, 5'-GTT GAC ATT CAA TCA AGC TGC-3'(sense) and 3'-CCA GTG GAT CTT TTT CCA GAG -3'(antisense) and htid-S, 5'-CAG CCT CAG GAA GAA ACC ATC-3'(sense) und 5'-GGG ATC GTC ACG TTG ATC GTC-3' (antisense) according to reaction conditions as described previously [13]. For the amplification of an HER-2 [16] specific fragment, encompassing nt 1850-2157 of the corresponding cDNA (NCBI reference sequence NM_001005862.1; $\underline{\text { http:// }}$ www.ncbi.nlm.nih.gov/sites/entrez) the primers 5'-CTC TGC TTC GTG CAC ACG GTG-3' (sense) and 5'-CAG GTC ACT GAG CCA TTC TGG-3' (antisense) (Eurofins MWG Operon, Ebersberg, Deutschland) were used at an annealing temperature of $60^{\circ} \mathrm{C}$. The relative expression level $(\Delta \mathrm{Ct})$ of a specific transcript was calculated with respect to the internal standard hypoxanthine guanine phosphoribosyl transferase (HGPRT) used in each reaction run to normalize variances in the quality of RNA, sample loading and the amount of input cDNA. Amplification of HGPRT was performed using the primers 5'TGA CAC TGG CAA AAC AAT GCA-3' (sense) and 5'GGT CCT TTT CAC CAG CAA GCT-3' (antisense) at an annealing temperature of $62^{\circ} \mathrm{C}$. Quantitative real-time RT-PCR analysis was performed using the ABI PRISM 7300 Sequence Detection System instrument and software (Applied Biosystems). The analysis of relative target expression was performed using the $2^{-\Delta \Delta C t}$ method [19]. To monitor DNA synthesis the fluorescent dye SYBRGreen was used. The cycle number at which the amplification of the transcript of interest was first detected is referred to as the cycle threshold, the $\mathrm{Ct}$ value. The 
increase in the fluorescence signal depends on the amount of the DNA in the starting PCR sample. The higher the DNA concentration, the faster a significant increase in fluorescence resulting in a low $\mathrm{Ct}$ value. The $\mathrm{Ct}$ value is proportional to the logarithm of the initial amount of the target DNA in the sample. The relative concentration of one target with respect to another is reflected in the difference of the cycle number, the $\Delta \mathrm{Ct}$ value. In this study the differences in the $\mathrm{Ct}$ values between the target gene/splice form investigated $(\mathrm{X})$ and the reference ( $R$, here HGPRT) are referred to as $\Delta C t$ values and were calculated as follows: $\Delta \mathrm{Ct}(\mathrm{X})=\mathrm{Ct}(\mathrm{X})$ $\mathrm{Ct}(\mathrm{R})$. A $\Delta \mathrm{Ct}=0$ indicates a ratio of 1 between the target and the reference $\left(1=2^{0}=2^{-(\Delta \mathrm{Ct})}\right)$. The factor 2 in the formula describes doubling of the fluorescence, the $\mathrm{Ct}$ value, at each cycle during the exponential phase of the PCR with $100 \%$ efficiency (E). Since $\mathrm{E}$ is determined by the RTPCR conditions, the E values were optimized for each target gene investigated prior to the sample analysis. For the $\Delta C t$ calculation to be valid, the efficiency of amplification of both the target and the reference must be approximately equal. The $\mathrm{E}$ values determined were as follows: $\mathrm{E}_{\mathrm{HGPRT}}=0,955 ; \mathrm{E}_{\mathrm{h} t i d-\mathrm{L}}=0,923 ; \mathrm{E}_{\mathrm{h} t i d-\mathrm{I}}=0,943 ; \mathrm{E}_{\mathrm{h} t i d-\mathrm{S}}=$ 0,$926 ; \mathrm{E}_{\text {erbB-2/her-2 }}=0,906$. The $\mathrm{E}$ values were calculated using the equation: $\mathrm{E}=10^{(-1 / \text { slope })}$. The $\mathrm{Ct}$ values versus cDNA concentration input were plotted to calculate the slope (mean \pm SD). Regressions were calculated using the graphad prism software as described previously [13]. The expression levels of the transcripts investigated in tumor samples were normalized to the respective normal tissues using $\Delta \Delta \mathrm{Ct}$ calculation as described previously [13]. The reported RNA expression levels represent the mean values $(\mathrm{n}=3) \pm$ standard deviation (SD). After standardization of both tumor and normal samples with respect to HGPRT, the change, $\Delta \Delta \mathrm{Ct}$, in the expression levels of the target transcripts in the tumor sample as compared to normal was calculated as follows: $\Delta \Delta \mathrm{Ct}=\Delta \mathrm{Ct}_{\text {tumor sample }}$ $\Delta \mathrm{Ct}_{\text {normal sample }}$

\section{Statistical examination}

Statistical evaluation of the RT-PCR data was performed using the one-sided Student's t-test.

For the statistical analysis of the correlation of the expression of the two target antigens determined by immunohistochemistry the Fischer's exact test was employed.

\section{Results}

The expression of htid and HER-2 RNA is altered in human breast tumors

The htid tumor suppressor gene encodes three splice forms - htid-L, htid-I and htid-S - generated by alternative splicing $[1,7,13]$. To determine the splice variant specific expression of the distinct htid transcripts in normal breast epithelium and in breast cancers, we performed quantitative RT-PCR analysis (Table 1 and 2). In the context of the suppressive activity of hTid proteins on ErbB-2 [15] the samples were further investigated for the expres-

Table 1: Quantification of the relative amounts of the three htid splice forms $L, I$ and $S$, and the $H E R-2$ transcript in human normal breast epithelium

\begin{tabular}{|c|c|c|c|c|}
\hline Target gene/splice form & htid-L & htid-I & htid-S & erbB-2 \\
\hline \multirow[t]{5}{*}{ Samples/ $\Delta \mathrm{Ct}$-value } & 4,11 & 1,54 & 15,82 & $-2,75$ \\
\hline & 3,17 & 0,59 & 15,38 & $-1,96$ \\
\hline & 3,53 & 0,57 & 13,69 & $-2,26$ \\
\hline & 3,78 & 0,84 & 14,95 & \\
\hline & 4,12 & 1,51 & 14,87 & \\
\hline average $\Delta \mathrm{Ct}(=100 \%)$ & 3,74 & 1,01 & 14,94 & $-2,32$ \\
\hline $\left.1 \Gamma_{2} \bar{x}\right)$ & $\pm 0,3(8,00 \%)$ & $\pm 0,4(39,61 \%)$ & $\pm 0,5(3,35 \%)$ & $\pm 0,29(12,50 \%)$ \\
\hline $\begin{array}{l}\text { Amount of target: } \\
\qquad 2^{-\Delta C t}\end{array}$ & 0,07 & 0,50 & $3.18 \times 10^{-5}$ & 4,99 \\
\hline
\end{tabular}

The $\Delta \mathrm{Ct}$ value is determined by substracting the HGPRT (endogenous reference) Ct-value from the $\mathrm{Ct}$ value of the target investigated: $\Delta \mathrm{Ct}=$ Ct (target) - Ct (HGPRT); Standard deviation (SD): $\left(\frac{1}{n} \sum \chi-\bar{\chi}\right), \mathrm{n}=3$, was calculated for the average $\Delta \mathrm{Ct}$ (100\%); The relative concentration of the target (amount of target), $2^{-\Delta C t}$, is calculated for the average $\Delta C t$ values. 
Table 2: Relative expression of the htid splice forms $L, I, S$ and the HER-2 transcript in human breast cancer

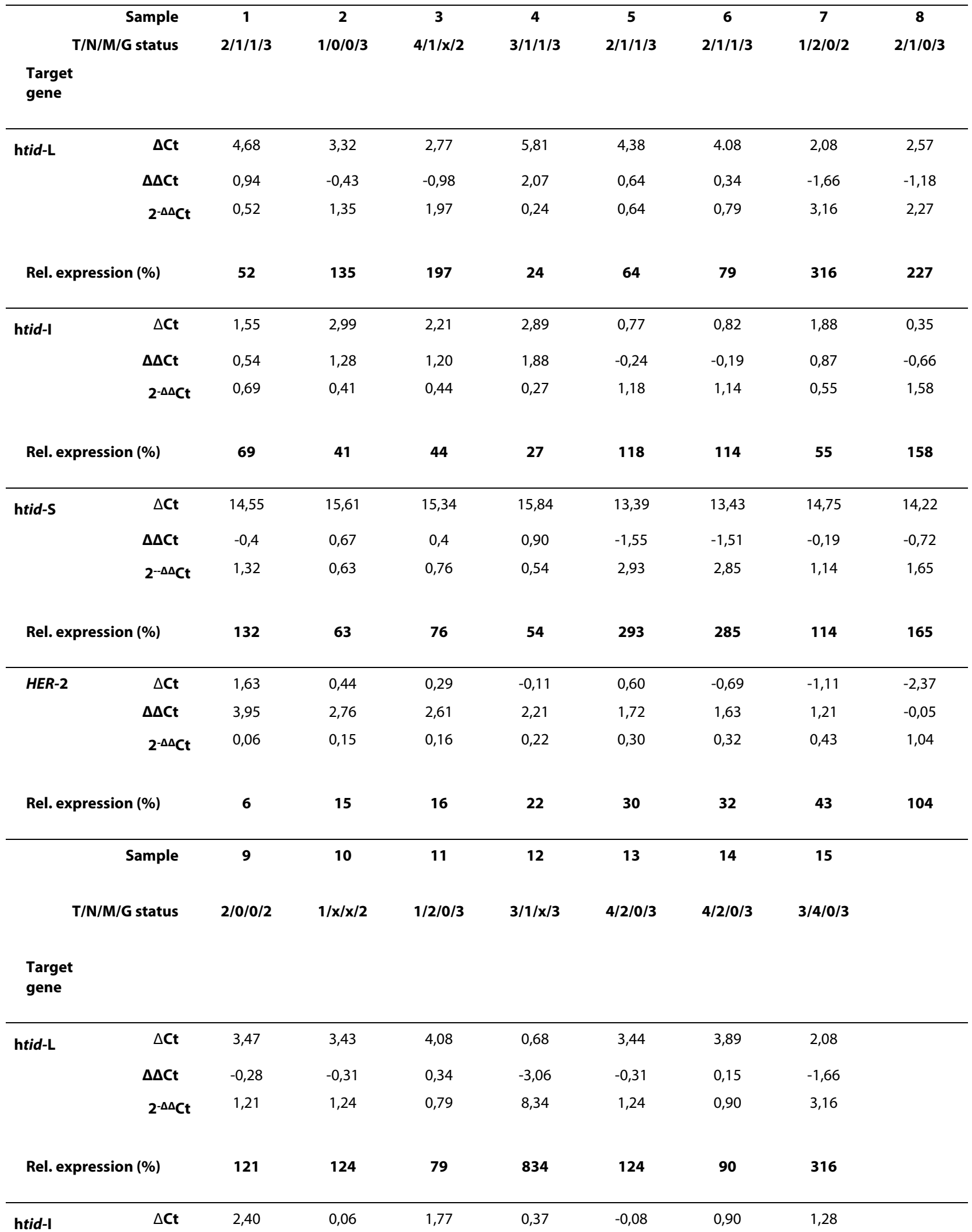


Table 2: Relative expression of the htid splice forms $L, I, S$ and the $H E R-2$ transcript in human breast cancer (Continued)

\begin{tabular}{|c|c|c|c|c|c|c|c|c|}
\hline & $\Delta \Delta C t$ & 1,39 & $-1,06$ & 0,76 & $-0,64$ & $-1,09$ & 0,11 & 0,27 \\
\hline & $2^{-\Delta \Delta} \mathrm{Ct}$ & 0,38 & 2,08 & 0,59 & 1,56 & 2,13 & 0,93 & 0,83 \\
\hline \multicolumn{2}{|c|}{ Rel. expression (\%) } & 38 & 208 & 59 & 156 & 213 & 93 & 83 \\
\hline \multirow[t]{3}{*}{ htid-S } & $\Delta \mathbf{C t}$ & 15,91 & 13,02 & 13,90 & 13,84 & 13,32 & 17,55 & 13,51 \\
\hline & $\Delta \Delta C_{t}$ & 0,97 & $-1,92$ & $-1,04$ & $-1,10$ & $-1,62$ & 2,61 & $-1,43$ \\
\hline & $2-\Delta \Delta C t$ & 0,51 & 3,80 & 2,06 & 2,14 & 3,07 & 0,16 & 2,70 \\
\hline \multicolumn{2}{|c|}{ Rel. expression (\%) } & 51 & 380 & 206 & 214 & 307 & 16 & 270 \\
\hline \multirow[t]{3}{*}{ HER-2 } & $\Delta \mathbf{C t}$ & $-2,63$ & $-2,97$ & $-3,00$ & $-3,23$ & $-3,40$ & $-5,71$ & $-6,31$ \\
\hline & $\Delta \Delta C_{t}$ & $-0,31$ & $-0,65$ & $-0,68$ & 0,91 & $-1,08$ & $-3,39$ & $-3,99$ \\
\hline & $2^{-\Delta \Delta} \mathrm{Ct}$ & 1,24 & 1,57 & 1,60 & 1,88 & 2,11 & 10,48 & 15.93 \\
\hline \multicolumn{2}{|c|}{ Rel. expression (\%) } & 124 & 157 & 160 & 180 & 211 & 1048 & 1593 \\
\hline
\end{tabular}

The samples are sorted according to the increase of HER-2 expression. The average $\Delta \mathrm{Ct}$ values $(\mathrm{n}=3)$ are presented. The calculation of $\Delta \Delta \mathrm{Ct}$ involves substraction by the $\Delta \mathrm{Ct}$ calibrator value (normal sample), $\Delta \Delta \mathrm{Ct}=\Delta \mathrm{Ct}$ (tumor sample) - $\Delta \mathrm{Ct}$ (normal sample). The SD of $\Delta \Delta \mathrm{Ct}$ is the same as the SD of the $\Delta \mathrm{Ct}$. The $\Delta \mathrm{Ct}$ values of both the tumor and normal samples are standardized to HGPRT used as endogenous control. The values defined as increase of expression represent the relative (rel.) change/increase in expression of the defined target as compared to normal sample and are calculated as follows: $2^{-\Delta \Delta C t} \times 100 \%$. T, tumor extension: $T 1$, to submucosa; $T 2$, to muscle layer; $T 3$, to subserosa; $T 4$, to serosa. $\mathrm{N}$, lymph node affection; $\mathrm{M}$, metastasis; $\mathrm{G}$, grading: $\mathrm{G} 1$, well differentiated; $\mathrm{G} 2$, moderately differentiated; $\mathrm{G} 3$, poorly differentiated; $\mathrm{x}$, not defined.

sion of the corresponding transcript. The evaluation of the amount of the targets investigated was performed using the comparative $\mathrm{Ct} / \Delta \Delta \mathrm{Ct}$ method (cf. Materials and Methods). As shown in Table 1 in normal breast epithelium the three htid splice forms are expressed at distinct levels. The $2^{-\Delta \mathrm{Ct}}$ values (Table 1 ) indicate highest expression for the $\mathrm{h} t i d-I$ form $(2-\Delta \mathrm{Ct}=0,50)$ and the lowest $\left(2^{-\Delta \mathrm{Ct}}=3,18 \times 10^{-5}\right)$ for the htid-S form. Generally, this expression profile corresponds to that we described previously for normal colon epithelium [13]. The expression level of the HER-2 transcript $(2-\triangle \mathrm{Ct}=4,99)$ in the breast epithelium samples which were defined as normal is higher (tenfold) as compared to the htid-I RNA (Table 1). As shown in Table 2, all breast tumors investigated express aberrant RNA levels of all htid splice forms as compared to normal tissue, independently from the level of the HER-2 transcript detected. With regard to the relative change of expression of the latter in the tumor samples as compared to normal the tumors can be divided into three groups. Whereas the first group is characterized by HER-2 levels ranging between 6-43\% (samples 17) as compared to normal (100\%), the third class, consisting of samples 14 and 15, shows drastic elevation (1048\% and 1593\%). The second group (samples 9-13) show slight to moderate increase of $H E R-2$ expression ranging from
$124 \%$ to $211 \%$. One case, 8 , shows similar $H E R-2$ level as that detected in normal sample (Table 1 ). Comparing the TNMG status of the samples and the htid expression profiles implicates that aspects of tumor progression such as tumor extension $(\mathrm{T})$, lymph node affection $(\mathrm{N})$ and loss of differentiation (G) are accompanied by elevation of htid-L level (Table 2, cases: 3,7,8,12,13,15) ranging from 124 to $834 \%$. In contrast, poorly differentiated and undifferentiated cases are characterized by decrease of $\mathrm{h} t i d-\mathrm{L}$ (Table 2, cases: 1, 4-6 and 11). With regard to the expression of the htid-I form, the cases can be divided into two groups, those which show lower expression as compared to normal (Table 2, cases: $1-4,7,9,11,14,15$ ) and those with levels elevated up to twofold (Table 2, cases: 5, 6, 8, 10, 12 and 13). Similarly, htid-S expression in the tumors is either down regulated in a range from $76 \%$ to $16 \%$, (Table 2, cases: 2-4, 9 und 14) or increased in a range from $114 \%$ to $380 \%$ (Table 2, cases: $1,5-8,10-13$ and 15 ). With respect to the two latter forms, $\mathrm{h} t i d-\mathrm{I}$ and $\mathrm{h} t i d-\mathrm{S}$, no correlation to the TNMG status is recognizable. Generally, the deregulation of the expression levels of the single $\mathrm{h} t i d$ transcripts leads to a collapse of the concentration ratio among the single $\mathrm{h} t i d$ forms and between the $\mathrm{h} t i d$ variants and the $H E R-2$ transcript. 


\section{Expression of htid in breast tumors as revealed by immunohistochemistry}

To obtain an overall assessment of $\mathrm{htid}$ expression in normal and transformed mammary epithelium we submitted to immunohistochemical analysis morphologically normal breast tissue (3 cases), random selected infiltrating ductal carcinomas (75 cases) and metastatic lesions (30 cases). Staining of these substrates with the affinity purified hTid antiserum $[6,7,13]$ demonstrated focal expression of the chaperone molecule only in the epithelial component of morphologically normal breast (Figure 1A, insert). In contrast about $43 \%$ of primary and $30 \%$ of metastatic tumors (including lymphonodal and extra-nodal lesions) were characterized by positive staining ranging from moderate to intense staining.

In the context of the potential association of htid expression with the biology of human breast cancers of different subtype the immunohistochemical analysis revealed elevated htid expression in $81 \%$ of the luminal A type tumors (Figure 1A), in $20 \%$ of the cases of the luminal B type over expressing ErbB-2 and in 18\% of tumors diagnosed as the HER-2 subtype (not shown). Tumors of the triple negative type showed positive staining for antiTid in $40 \%$ of the cases investigated (Figure 1B-D).

\section{Correlation between $\mathrm{htid}$ and ErbB-2 expression in breast and non-breast tumors over expressing the ErbB-2 receptor as revealed by immunohistochemistry}

In view of the above findings which are consistent with the in vitro findings of hTid oncosuppressive activity on ErbB-2 [15], we focussed our further investigation on the analysis of the in vivo correlation between htid and HER2 expression. Regarding the expression of the two target genes the following patterns were compared: double positive $(H E R-2+/$ tid +$)$, double negative $(H E R-2-/$ tid -$)$ and positive/negative (HER-2 +/tid -; tid +/HER-2 -). Specimens characterized by lack or faint expression of the tar-
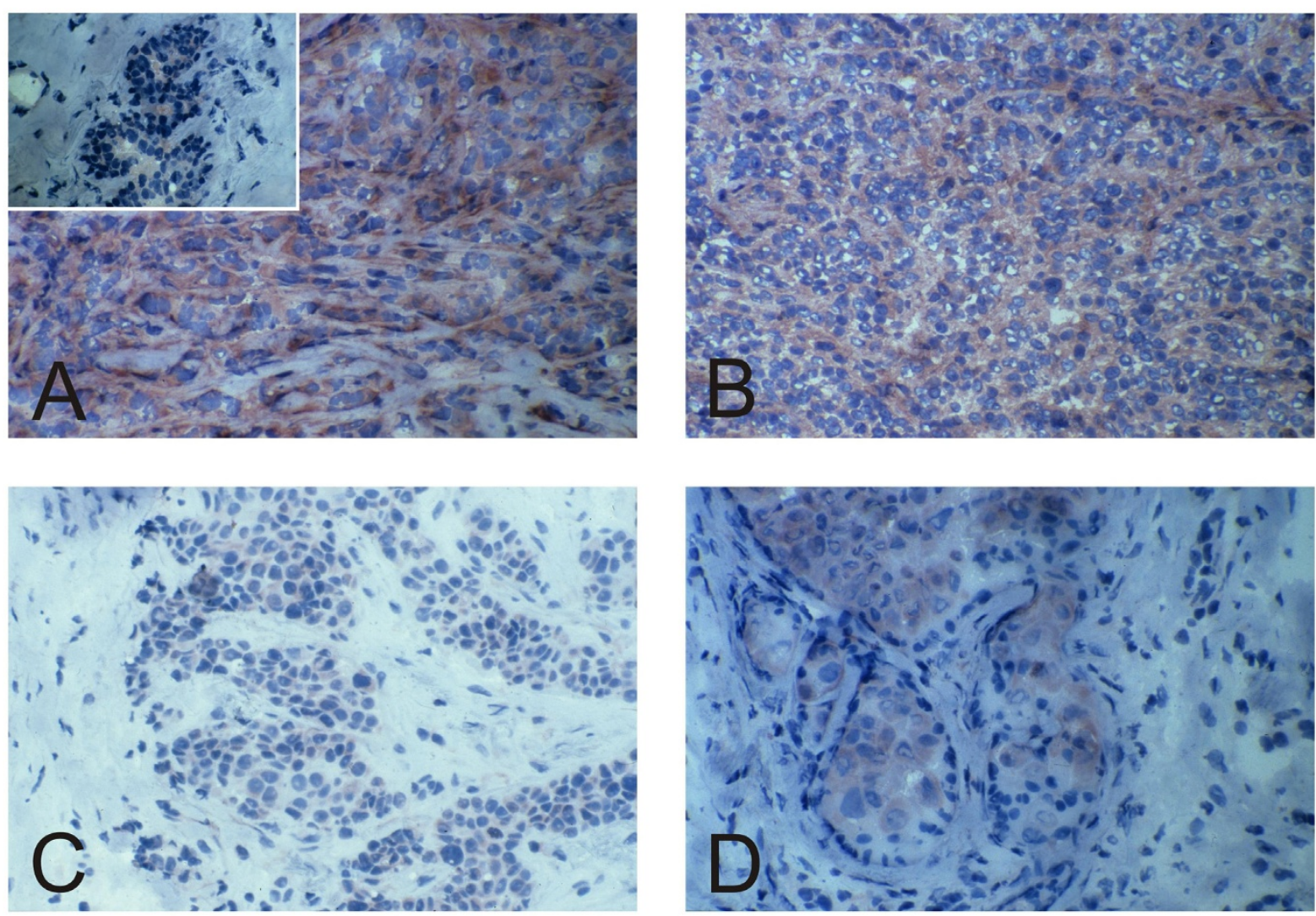

Figure 1 Representative expression patterns of htid in normal mammary epithelium and in primary breast tumors of different subtype. Indirect immunoperoxidase staining using the Vectastain ABC Kit was performed according to manufacturer's suggestions using the polyclonal rabbit anti-hTid $(6,7,13)$. Nuclei were counterstained with Mayer's hematoxylin. While htid is expressed at low levels in the normal breast epithelium (A: insert) its expression is significantly elevated in the luminal $A(A)$ and $B(B)$ tumor type. In contrast, htid is barely detectable in tumors of the HER-2 (C) and the triple negative (D) subtype. (Original magnification: 250x). 
get antigens were defined as negative. Samples showing moderate $(+)$ to strong $(2+/ 3+)$ signals by staining them with anti hTid antibodies and $3+$ or $2+/$ Fish + stain using antibodies against ErbB-2 were defined as positive. In that context we assayed comparatively the expression levels of the proteins encoded by the two target genes in 24 primary tumors over expressing the receptor (17 of the HER-2 and 7 of the luminal B subtype) and 34 ErbB-2 negative tumors (16 luminal A, 8 luminal $B$ and 10 of the triple negative subtype) as well as in metastatic lesions over expressing the TRK receptor by staining with antiTid $[6,7,13]$ and antibodies against ErbB-2. The results of this analysis are summarized in Table A3A and Figure 2A-D. They clearly demonstrate that the expression of the two targets investigated is inversely correlated in both primary $(\mathrm{p}<$ in 0,0001$)$ and in metastatic $(\mathrm{p}<0.023)$ mammary tumors (not shown). Since ErbB-2 over expression may occur also in other epithelial cancers [18], we performed a comparative staining of HER-2 and htid in non mammary carcinomas characterized by elevated levels of ErbB-2 (10 cases) and in cancers displaying low levels of the receptor (8 cases). This study (Table B3B) also yielded a highly significant inverse correlation $(\mathrm{p}<$ 0,007 ) with respect to the expression levels of the two tumor genes investigated (Figure 2E, F).

\section{Correlation between htid and ErbB-2 expression in breast tumors induced in Her-2/neu transgenic mice}

As described above, the inverse correlation of the expression profiles of the two proteins in human breast tumors and non mammary malignancies provides an in vivo proof for the oncosuppressive activity of htid on ErbB-2 described in vitro [15]. Furthermore, since the inverse relationship is highly significant in diverse tissues, this functional link is not cell specific, and therefore of general biological importance. Next, we asked whether the inverse correlation is evolutionarily conserved in the mouse. To answer this question we employed the transgenic mice model of Her-2/neu induced breast tumors [23]. We stained early (22 weeks) and late (30 weeks) breast tumors generated in transgenic mice carrying the rat Her-2/neu oncogene. As shown in Figure 3A, staining of the early tumors with the hTid [7,13] antiserum revealed homogenous $(1+)$ cytoplasmic expression. Dif- ferently, using the anti-ErbB-2 antiserum cross reacting with the murine receptor resulted in a faint stain of the cytoplasm and rarely in staining of the membrane of the tumor cells (Figure 3B). In the more advanced 30 weeks tumors the staining patterns changed with regard to both the expression level and distribution. In these lesions, the hTid expression was heterogeneous with alternating areas of moderate to intense $(2+)$ cytoplasmic stain (Figure $3 \mathrm{C}$ ). The expression of ErbB-2 also appeared heterogenous with only discrete areas of the tumor displaying an intense $(2+)$ staining often cell membrane associated (Figure 3D). This result further suggested that the inverse correlation of the expression levels of the two molecules is indeed detectable also in experimental tumors raised in mice carrying the rat $\mathrm{Her}-2 / \mathrm{neu}$ oncogene. In order to conclusively prove this issue, we submitted the more advanced tumors to double staining using anti-hTid $[5,13]$ and the chicken antibody recognizing the murine TRK receptor molecule. As shown in Figure 4, tumor areas characterized by intense membrane ErbB-2 expression (A) display significantly lower htid level $(\mathbf{B}, \mathbf{C})$, thus, demonstrating the inverse correlation of the expression of the two tumor relevant molecules and implying their functional link.

\section{Discussion}

The understanding of the mechanism responsible for the in vivo oncosuppressive action of htid in human tumorigenesis is of biological relevance in view of the multiple molecular interactions of the proteins it encodes [7$15,25,26]$. The ability of the hTid proteins to interact with distinct cancer related molecules, mediating via linked signal transduction networks diverse cellular processes, suggests that the deregulation of their expression may affect simultaneously diverse cellular functions. Furthermore, the identification of the htid encoded proteins as components of multi-component complexes suggests that their activity is likely to be associated with mechanisms determining the sequential and temporarily determined assembling of these complexes in the cell and their cellular topology. This is consistent with the function of molecules defined as chaperones and their molecular assistants known as co-chaperons. The Tid proteins $[1,2,6,7,13]$ are indeed members of the DNAJA3 family

Table 3: The expression of hTid and ErbB-2 is inversely correlated in primary breast cancer (A) and in non mammary tumors (B).

\begin{tabular}{cccccc}
\hline A 1 & hTid (+) & hTid (-) & B2 & hTid (+) & $2^{*}$ \\
\hline ErbB-2 (+) & $2^{*}$ & $22^{*}$ & ErbB-2 (+) & \\
\hline ErbB-2 (-) & $28^{*}$ & $6^{*}$ & ErbB-2 (-) & $8^{*}$ \\
\hline
\end{tabular}

${ }^{*}:$ number of cases; ${ }^{1}: p<0,0001 ;{ }^{2}: p<0,007 ;$ 

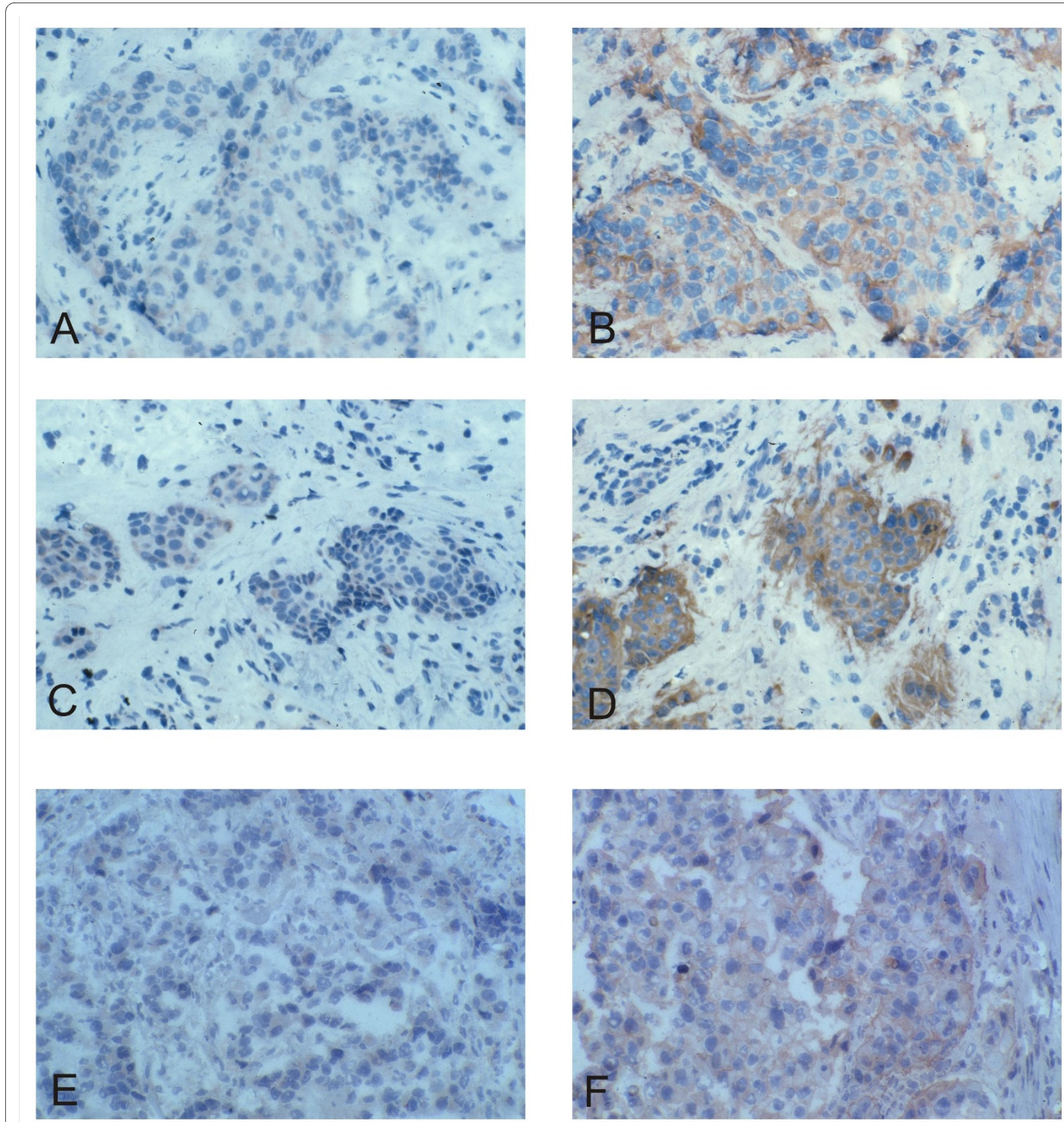

Figure 2 Comparative analysis of htid and HER-2 expression in breast and non breast tumors. The detection of htid was performed as described in the legend to Figure 1. The ErbB-2 oncogene was detected using a monoclonal anti ErbB-2 antibody (AO485). Weak htid expression is detectable in HER-2 over expressing breast tumors of the luminal $B(A, B)$ and HER-2 (C, D) subtype as well as in a renal clear cell carcinoma (E, F). (Original magnification: 250X).

encoding Hsp70/Hsc70 co-chaperone molecules [4,5]. The understanding of their role in the spatial and temporal organization of either active or silent protein complexes is of relevance in the context of both general developmental processes and, especially, tumor biology [6-15]. Regarding the latter the knowledge of the biological context these molecules are involved in may have an essential impact for the identification of novel causal cancer therapies.

Data are available indicating that the cytosolic hTidproteins $\mathrm{L}$ and I mediate cellular tumor-related processes such as proliferation, differentiation and migration by modulating/stabilizing signalling pathways driving these processes [6,7,9-14]. Generally, two modes of action of 

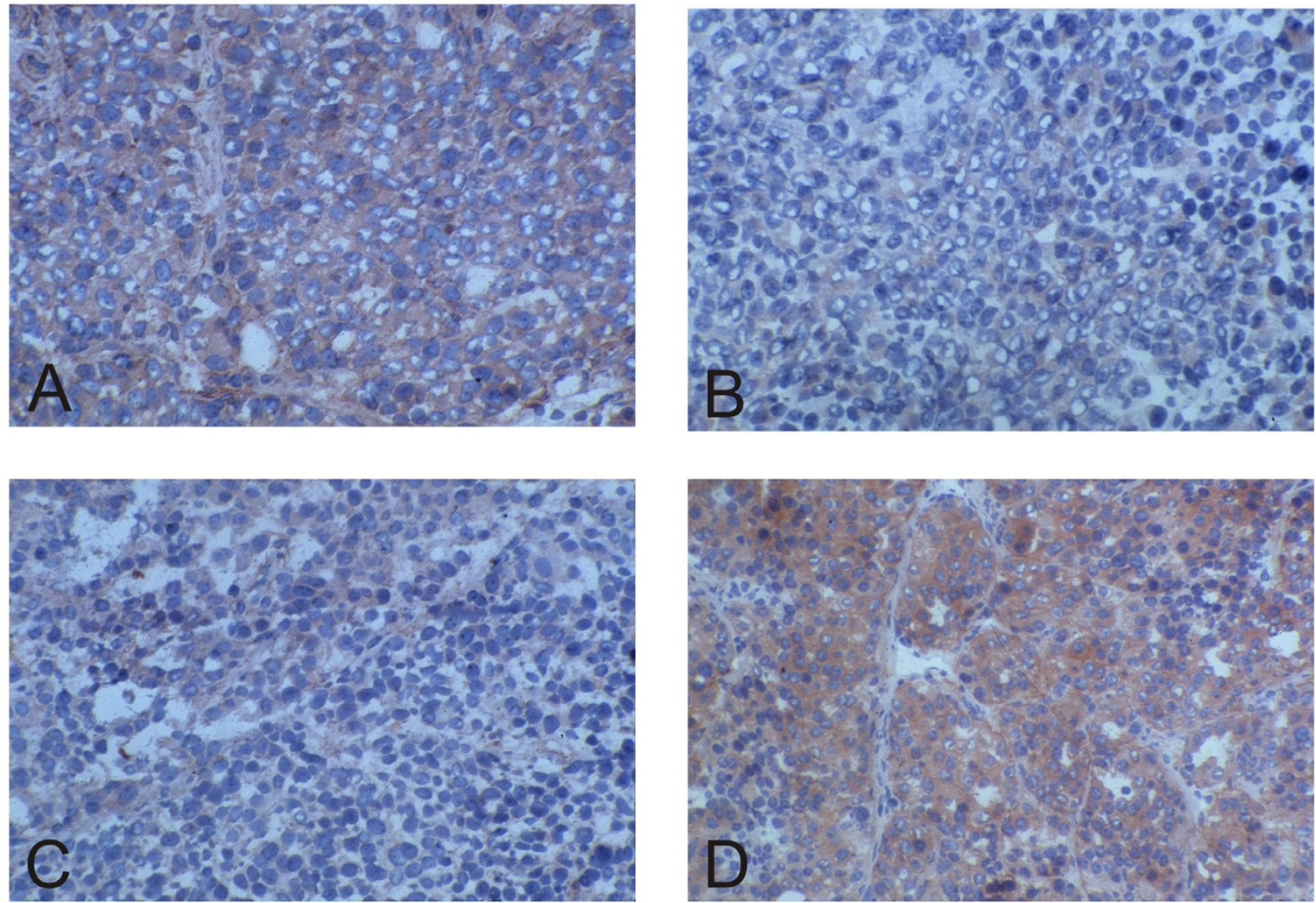

Figure 3 Comparative analysis of htid and HER-2/neu expression in breast tumors arising in transgenic Balb/c mice carrying the rat $H E R-2 /$ neu oncogene revealed inverse correlation between the expression levels of the investigated targets. The detection of mtid was performed as described in the legend to Figure 1. ErbB-2 was detected using the monoclonal anti ErbB-2 antibody (AO85) (cf. Figure 2) cross reacting with the rat molecule. While early tumors, 22 week old, display high tid (A) and low HER-2/neu levels (B), those harvested at 30 weeks are characterized by a low tid expression (C) in contrast to high HER-2/neu (D) levels. (Original magnification: 250x).

the Tid proteins can be discerned to date: i) interaction with cytosolic molecules, such as the APC tumor suppressor [7,13], a central component of the Wingless/Wnt pathway and the E-Cadherin mediated signalling or the Inhibitor of IKB in the NFKB signalling [11], crucial for driving the expression of regulators of cell cycle control, or ii) binding to receptors mediating the activation of signalling pathways, e.g. Ptc [6,7] and ErbB-2 [15]. With regard to the latter the regulatory functions of the cytosolic htid splice forms hTid-L and hTid-I have been shown to participate in the degradation of the receptor mediated by the Hsp70/CHIP ubiquitin ligase complex [15]. Interestingly enough, both hTid forms, L and I, are suggested to be equally capable to down regulate the over expression of the ErbB-2 receptor and to decrease its oncogenic signalling in human breast cancer cell lines in which the co-chaperone molecule was over expressed [15]. This observation strongly suggests that the ErbB-2 concentration level is relevant in activating the suppressive function of the two hTid proteins [15]. Furthermore, it raises the question whether the binding of the two hTid forms with ErbB-2 is functionally equal also under physiological circumstances. We asked these questions during the study presented here. Functional equality under physiological conditions suggests, as a result, that these two proteins may complement each others' function(s) under peculiar circumstances, e.g. pathological states.

Overall, the in vivo data of the present study further support the growing evidence that the anticancer activity of the hTid proteins occurs through the interference with multiple oncogenic pathways by binding and modifying functions of key molecules maintaining intracellular signalling. The ErbB-2 TRK represents one of these molecules.

As revealed by the RT-PCR analysis, the three htid splice forms are differentially expressed in normal breast epithelium. Their concentration levels are in this tissue similar to those we described previously for colon epithelium [13], the I form with the highest expression level and the $\mathrm{S}$ form characterized by very low concentration. This 

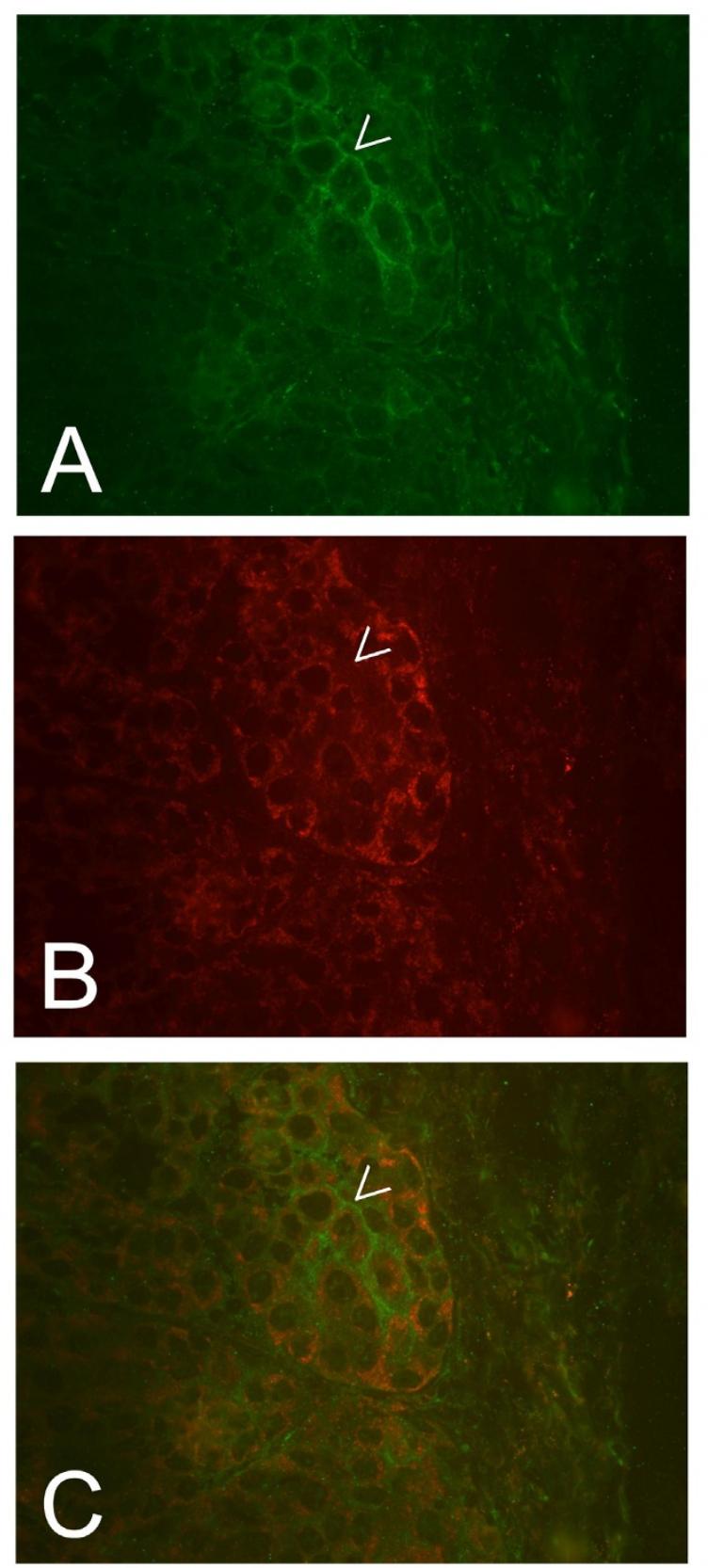

Figure 4 Photographs of simultaneous staining of 30 week old breast tumors arising in HER-2/neu transgenic mice illustrating that over expression of $H E R-2 / n e u$ correlates down regulation of htid. ErbB-2 was detected using a polyclonal chicken anti rat ErbB-2 and a FITC labelled secondary antibody. Tid was detected using the rabbit polyclonal anti hTid antibody $[6,7,13]$ and a Texas Red labelled secondary antibody. The tumor cells over expressing ErbB-2 (A, arrowhead) are characterized by low tid expression (B, arrowhead; cf. Figure 3). In $C$ an overlay of the photographs shown in $A$ and $B$ is presented.

suggests that under normal physiological conditions the concentration of the three htid splice forms is precisely regulated and that this mechanism is essential for the diverse functions the single molecules are maintaining in the cell. Previously we showed that the expression profile changes in epithelial tumors such as basal cell carcinomas (BCCs) [6] and colon cancers [13]. As shown here, this is also true for human breast tumors which are characterized by alteration of expression of all three htid forms resulting at least in a collapse of the concentration ratio between them. Since the hTid proteins are highly promiscuous [6-15] this phenomenon must, as a result, drastically affect the homeostasis of the cellular functions these complexes are involved in. Thus, the deregulation of htid leads independently of the causative event to severe cellular derangements. Additionally to this general conclusion a further more specific observation, concerning the aforementioned in vivo functional equality of the two forms $\mathrm{L}$ and $\mathrm{I}$, can be driven from the present study. The RT-PCR data demonstrate up-regulation of expression of the hTid-L form in most of the non metastasizing tumors, whereas poorly differentiated and undifferentiated cases with lymph node metastases show a decrease of the level of this form up to $24 \%$ of the normal (100\%) level. This pattern which suggests that hTid may exert oncosuppressive activity which breaks down during tumor progression is consistent with the role postulated for the L and I forms [15] by in vitro studies employing the ErbB-2 over expressing cell lines. Furthermore, the RT-PCR data indicate that the hTid-L oncosuppressive action may be of general importance being not limited to ErbB-2 over expressing malignancies. In view of this, one can hypothesize at the present the following model for the activation of this security/emergency mechanism: if the concentration of a molecule driving the tumorigenic process, e.g. ErbB-2, riches a threshold level hTid-L expression is enhanced. This rescuing mechanism however is time limited as hTid expression declines during tumor progression. This hypothesis raises questions concerning the mechanisms regulating the physiological and emergency expression of htid. The answer to this question is the subject of our present investigations. So far we can state that consistent with the molecular promiscuity of the hTid proteins distinct cell specific and cell status dependent regulatory mechanisms are involved in these processes. As previously mentioned, the functional equality of $\mathrm{L}$ and I under physiological circumstances would suggest that these two forms may complement each others function under specific circumstances. Under the assumption that this is true a shift of the concentration ratio of the two forms could be expected as a consequence of their oncosuppressive/protective function on the tumorigenic targets. Though this hypothesis is from the mechanistic point of view very attractive, the analysis of the correlation of the expression levels of the two forms $\mathrm{L}$ and I in the tumor samples do not support it at present. Decrease in the level of the $\mathrm{L}$ form do not correspond to increase of the concentration of the I form and vice versa. Thus, to solve the problem of the functional equality of the two 
hTid forms L and I further investigations using adequate in vivo methodology are necessary. With regard to the htid splice forms I and S, which expression levels are also changed in nearly all tumor samples investigated, no correlation with the TNMG status and the HER-2 level could be found.

The immunohistochemical analysis described in this study provided additional interesting findings. It showed that the changes in the expression profiles of the targets investigated during tumor progression of experimental tumors are not ubiquitous but rather hot-spot like. This is consistent with the fact that functional complexes including the Tid proteins are built in the cells sequentially and are topologically determined as we discussed previously $[6,7]$. Most importantly, the data provide in vivo evidence that $\mathrm{h}$ tid is a potential modulator of ErbB-2 signalling in both breast and non-breast tumors over expressing the kinase. Furthermore, since the inverse correlation of the expression levels of the two genes is highly significant in human breast cancers as well as in experimental breast tumors the functional relationship between the two targets is conserved. Furthermore, the transgenic mice animal model may be suitable to in vivo dissect the oncosuppressive activity of the hTid proteins on ErbB-2 function. In view of the association of ErbB-2 over expression with poor prognosis in breast cancers the elucidation of these phenomena is of particular biological interest also in the context of the pharmacological relevance of ErbB-2 regulation, which, possibly, could be modulated via tuning of expression of the single htid splice forms. With regard to the latter reduction of htid expression can in fact render cancer cells resistant to apoptosis induced by a wide spectrum of stimuli including TNF- $\alpha$ [25] and increase their migratory potential [26].

The variety of biological processes the EGFR family members regulate results from the differential signalling they mediate by building both homo and diverse heterodimers between the family members responding to distinct ligands [16,27-29]. ErbB-2 is the favourite partner of all other EGFR receptors [29]. The ErbB-2/ErbB-3 heterodimer is a high affinity receptor for neuregulins endowed with potent oncogenic activity [28]. In view of this the hTid proteins can also be considered as potential EGFR and/or HER-3 binding partners and therefore players in the signalling they induce. In this context a preliminary comparative expression analysis of hTid, EGFR and ErbB-3 we have conducted in triple negative breast tumors and in cutaneous melanoma (data not shown) respectively also suggests an inverse correlation between the expression levels of hTid and the two receptors, thus, indicating that $\mathrm{htid}$ functional relation may indeed encompass other members of the EGFR family. Since these receptors control different developmental pro- cesses their activation/deactivation requires a fine tuning. The on status is activated by paracrine and autocrine signals engaging one or more receptors [30]. The off status is strengthened by a number of feedback inhibitors [30]. So far four ErbB-2 inhibitors have been described, namely, LRIG1 [31], SOCSS4 and 5 [32] and RALT/MIG6 [33,34]. While the first three inhibitors interfere temporary with EGFR activity by enhancing its ubiquitination, RALT is endowed with the ability to block the ErbB RTK signalling by its inception [33]. Our in vivo results suggest hTid$\mathrm{L}$ as a further modulator of ErbB-2 activity in human tumors of epithelial origin. In accordance with the reported molecular analysis in vitro [15] it can be postulated that this may occur via mechanisms similar to those described for the negative feedback inhibitors LRIG1 and SOCS4 and 5. This hypothesis has to be confirmed using appropriate experimental approaches.

\section{Conclusions}

In summary, the presented data provide in vivo evidence for htid function as a negative regulator of ErbB-2 activity. The inverse correlation of the expression levels of the two genes is highly significant in human breast and nonmammary cancers, and in experimental tumors raised in transgenic mice carrying the rat HER-2/neu oncogene. Thus, the functional relationship between the two targets is tissue independent and evolutionarily conserved.

\section{Competing interests}

The authors declare that they have no competing interests.

\section{Authors' contributions}

UKD designed and evaluated the RT-PCR experiments, generated and purified the anti-hTid antibody and drafted the manuscript. $\mathrm{MH}$ determined htid expression via RT-PCR. MRN performed the immunohistochemistry of human and murine carcinomas. MK determined HER-2 expression via RT-PCR. PGN designed and analyzed the immunohistochemical data and drafted the manuscript. All authors read and approved the final manuscript.

\section{Acknowledgements}

This work was supported in part by the German Cancer Society (grant 10-1695Ku2 to UK-D) and by Associazione Italiana Ricerca sul Cancro (AIRC) (grant to PGN).

We would like to thank Prof. Forni, Dept. of Clinical and Biological Sciences, University of Turin, Italy, for providing the breast tumors developed in transgenic Balb/c mice carrying the rat HER-2/neu oncogene and Dr. Ö Türeci, Experimental and Translational Oncology III, University Medical Center, Johannes Gutenberg University, Mainz, Germany, for providing the tissue samples used for RTPCR analysis.

\section{Author Details}

${ }^{1}$ Institute of Medical Microbiology and Hygiene, Comparative Tumor Biology Group, University Medical Center, Johannes Gutenberg University, Obere Zahlbacher Str. 63, 55131 Mainz, Germany, 2Inst. Molecular Biology and Pathology CNR, Rome, Italy, ${ }^{3}$ Experimental and Translational Oncology III, University Medical Center, Johannes Gutenberg University, Obere Zahlbacher Str. 63, 55131 Mainz, Germany and 4Immunology Laboratory, "Regina Elena" National Cancer Institute, Via delle Messi d'Oro 156, 0158 Rome and CIMBO Laboratories, "G.d'Annunzio" University, Chieti, Italy

Received: 9 April 2010 Accepted: 17 June 2010

Published: 17 June 2010 


\section{References}

1. Yin X, Rozakis-Adcock M: Genomic organization and expression of the human tumorous imaginal disc (TID1) gene. Gene 2001, 278:201-210.

2. Kurzik-Dumke U, Gundacker D, Rentrop M, Gateff E: Tumor suppression in Drosophila is causally related to the function of the lethal(2)tumorous imaginal discs gene, a dna J homolog. Dev Genetics 1995, 16:64-76.

3. Ohtsuka K, Hata M: Molecular chaperone function of mammalian Hsp70 and Hsp40- review. Int J Hyperthermia 2000, 16:231-2452.

4. Qiu X-B, Shao Y-M, Miao S, Wang L: The diversity o the DnaJ/Hsp40 family, the crucial partners for Hsp70 chaperones. Cell Mol Life Sci 2006, 63:2560-2570.

5. Vos MJ, Hageman J, Carra S, Kampinga HH: Structural and functional diversities between members of the human HSPB, HSPH, HSPA and DNAJ chaperone families. Biochemistry 2008, 47:7001-7011.

6. Canamasas I, Debes A, Natali PG, Kurzik-Dumke U: Understanding Human Cancer Using Drosophila: Tid47, a cytosolic product of the DnaJ-like tumor suppressor gene I(2)tid, is a novel molecular partner of Patched related to skin cancer. J Biol Chem 2003, 278:30952-30960.

7. Kurzik-Dumke U, Czaja J: Htid-1, the human homolog of the Drosophila melanogaster I(2)tid tumor suppressor, defines a novel physiological role of APC. Cellular Signaling 2007, 18:1973-1985.

8. Schilling B, De-Medina T, Syken J, Vidal M, Münger K: A novel human DnaJ protein, hTid- 1 homolog of the Drosophila tumor suppressor protein Tid56, can interact with the human papillommavirus type 16 E7 oncoprotein. Virology 1998, 247:74-85.

9. Sarkar S, Pollack BP, Lin K-T, Kotenko SV, Cook JR, Lewis S, Pestka S: HTid-1, a human DnaJ protein, modulates the interferon signalling pathway. $J$ Biol Chem 2001, 276:49034-49042.

10. Cheng H, Cenciarelli H, Shao Z, Vidal M, Parks WP, Pagano M, Cheng-Mayer C: Human T cell leukaemia virus type 1 Tax associates with a molecular chaperone complex containing hTid-1 and Hsp70. Current Biol 2001, 11:1771-1775

11. Cheng H, Cenciarelli C, Tao M, Parks WP, Cheng-Mayer C: HTLV-1 Taxassociated $\mathrm{hTid}-1$, a human DnaJ protein, is a repressor of IKB Kinase $\beta$ subunit. J Biol Chem 2002, 277:20605-20610.

12. Bae M-K, Jeong J-W, Kim S-H, Kim S-Y, Kang YJ, Kim D-M, Bae S-K, Yun I, Trentin GA, Rozakis-Adcock M, Kim K-W: Tid-1 interacts with the von Hippel-Lindau protein and modulates angiogenesis by destabilization of HIF-1a. Cancer Res 2005, 65:2520-2525.

13. Kurzik-Dumke U, Hörner M, Czaja J, Nicotra MR, Simiantonaki N, Koslowski $M$, Natali PG: Progression of colorectal cancers correlates with overexpression and loss of polarization of expression of the htid-1 tumor suppressor. Int J Mol Med 2008, 21:19-31.

14. Liu H-Y, MacDonald JIS, Hryciw T, Li Ch, Meakin SO: Human Tid1 associates with TRK receptor tyrosine kinases and regulates neurite outgrowth in NNR5-TRKA Cells. J Biol Chem 2005, 7:19461-19471.

15. Kim S-W, Chao T-H, Xiang R, Campbell MJ, Fearns C, Lee J-D: Tid1, the human homoloque of a Drosophila tumor suppressor, reduces the malignant activity of ErbB-2 in carcinoma cells. Cancer Res 2004 64:7732-7739

16. Citri A, Yarden Y: EGF-ERBB signalling: towards the system level. Nature Rev Molec Cell Biol 2006, 7:7505-7516.

17. Slamon DJ, Clark GM, Wong SG, Levin WJ, Ullrich A, McGuire WL: Human breast cancer: correlation of relapse and survival with amplification of the HER-2/neu oncogene. Science 1987, 235:177-182.

18. Agus BD, Bunn PA, Franklin W, Garcia M, Ozols RF: HER-2/neu as a therapeutic target in non small cell lung cancer, prostate cancer and ovarian cancer. Semin In Oncol 2000, 27:53-63.

19. Livak KJ, Schmittgen TD: Analysis of relative gene expression data using

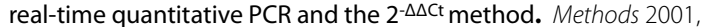
25:402-408

20. Sobin LH, Wittening CH: TNM Classification of Malignant Tumors J WilleyLiss, Willey Sons, Inc., New York; 2002.

21. Elston CW, Ellis IO: Pathological prognostic factors in breast cancer. I. The value of histological grade in breast cancer: experience from a large study with long-term follow- up. Histopathology 1991, 19:403-410,

22. Sotiriou C, Pusztai L: Gene-expression signature in breast cancer. New Engl J Med 2009, 360:790-800.

23. Nanni P, Nicoletti G, De Giovanni C, Landuzzi L, Di Carlo E, Cavallo F, Pupa SM, Rossi I, Colombo MP, Ricci C, Astolfi A, Musiani P, Forni G, Lollini P-L: Combined allogenic tumor cell vaccination and systemic interleukin
12 prevent mammary carcinogenesis in HER- 2 /neu transgenic mice. $J$ Exp Med 2001, 194:1195-1206.

24. Wolf-Yadiin A, Kumar N, Zhang Y, Hautaniemi S, Zaman M, Kim H-D, Grantcharova V, Lauffenburger DA, White FM: American Society of Clinical Oncology/College of American Pathologists guideline recommendations for human epidermal growth factor receptor 2 testing in breast cancer. J Clin Oncol 2007, 25:118-43.

25. Edwards K, Munger K: Depletion of physiological levels of the human TID1 protein renders cancer cell lines resistant to apoptosis mediated by multiple signalling. Oncogene 2004, 23:8419-8431.

26. Kim S-W, Hayashi M, LO J-F, Fearns C, Xiang R, Lazennec G, Xang Y, Lee J-D: Tid1 negatively regulates the migratory potential of cancer cells by inhibiting the production of interleukin-8. Cancer Res 2005, 65:8784-8791

27. Earp HS, Calvo BF, Sartor Cl: The EGF receptor family multiple roles in proliferation, differentiation, and neoplasia with an emphasis on Her-4. Trans Am Clin and Climatol Assoc 2003, 114:315-334.

28. Alimandi M, Romano A, Curia MC, Muraro R, Fedi P, Aaronson SA, Di Fiore $\mathrm{PP}$, Kraus MH: Cooperative signalling of ErbB3 and ErbB2 in neoplastic transformation and human mammary carcinoma. Oncogene 1995, 10:1813-182.

29. Graus-Porta D, Beerli RR, Daly JM, Hynes NE: ErbB-2, the preferred heterodimerization partner of all ErbB receptors is a mediator of lateral signaling. $E M B O J$ J 1997, 16:1647-1655

30. Fiorini M, Alimandi M, Fiorentino L, Sala G, Segatto O: Negative regulation of receptor tyrosine kinase signal. FEBS Lett 2001, 490:132-141.

31. Gur G, Rubin C, Katz M, Amit I, Citri A, Nilsson J, Amariglio N, Henriksson R, Rechavi G, Hedman H, Wides R, Yarden Y: LRIG1 restricts growth factor signalling by enhancing receptor ubiquitynation and degradation. EMBO J 2004, 23:3270-3281.

32. Kario E, Marmor MD, Adamsky K, Amit I, Amariglio N, Rechavi G, Yarden Y: Suppressors of cytokine signalling 4 and 5 regulate epidermal growth factor receptor signaling. J Biol Chem 2005, 280:7038-7048.

33. Fiorentino L, Pertica C, Fiorini M, Talora C, Crescenzi M, Castellani L, Alema S, Benedetti P, Segatto O: Inhibition of ErbB-2 mitogenic and transforming activity by RALT, a mitogen-induced signal transducer which binds to the ErbB-2 kinase domain. Mol Cell Biol 2000, 20:7735-7750

34. Anastasi S, Baietti MF, Frosi Y, Alemà S, Segatto O: The evolutionary conserved EBR module of RALT/MIG6, mediates suppression of the EGFR catalytic activity. Oncogene 2007, 26:78337849.

\section{doi: 10.1186/1479-5876-8-58}

Cite this article as: Kurzik-Dumke et al., In vivo evidence of htid suppressive activity on ErbB-2 in breast cancers over expressing the receptor Journal of Translational Medicine 2010, 8:58

\section{Submit your next manuscript to BioMed Central and take full advantage of:}

- Convenient online submission

- Thorough peer review

- No space constraints or color figure charges

- Immediate publication on acceptance

- Inclusion in PubMed, CAS, Scopus and Google Scholar

- Research which is freely available for redistribution
C) BioMed Central 